\title{
Impact of the Sloan Digital Sky Survey on Variable Star Research
}

\author{
Željko Ivezić ${ }^{1}$, Josh Goldston ${ }^{1}$, Kristian Finlator ${ }^{1}$, Jill Knapp ${ }^{1}$, Brian \\ Yanny $^{2}$ for the SDSS Collaboration
}

\begin{abstract}
We present the results of a preliminary search for variable objects in about $100 \mathrm{deg}^{2}$ of SDSS commissioning data surveyed twice with $\Delta t=1.99462$ days. In particular, we find that the density of RR Lyrae star candidates abruptly ends at a galactocentric radius of $50-60 \mathrm{kpc}$.
\end{abstract}

The Sloan Digital Sky Survey (SDSS) will be the first large-area photometric survey to utilize CCD detectors. Using a 2.5 -m wide-field special-purpose telescope, it will produce a detailed digital photometric map in five colors of half the northern sky to about $23 \mathrm{mag}$. About $225 \mathrm{deg}^{2}$ in a long, thin stripe along celestial equator will be scanned about 45 times. These scans will be co-added to produce a single, about 2 magnitudes deeper image, and differences between scans will be used to identify variable objects. In addition, because of the overlaps in scans, about $40 \%$ of the total covered area $\left(4000 \mathrm{deg}^{2}\right)$ will be observed twice. Although two observations are hardly enough to characterize a variable object, with the help of 5-band photometric data SDSS is expected to vastly increase the known number of variable stars.

Here we present the results of a preliminary search for variable objects in about $100 \mathrm{deg}^{2}$ of sky surveyed twice with $\Delta t=1.99462 \mathrm{~d}$. About $0.05 \%$ of the point sources show significant variation: most of these are either flare stars or variable objects with $A$-star colors and thus probably dominated by $R R$ Lyrae stars. Assuming the latter to be true, we derive the volume density of RR Lyrae stars from the observed mean $r^{\prime}$ magnitude distribution. Our results show two important differences when compared to previous work: the density of RR Lyrae stars follows a much shallower power law $(2.3 \pm 0.2)$ than conventional power law $\mathrm{r}^{-3}$, and it abruptly ends at a galactocentric radius of $50-60 \mathrm{kpc}$. While these results are based on a preliminary search of a small area and are sensitive to contamination by variable stars other than RR Lyrae stars, they can be easily verified when the light curves for all candidates become available.

\footnotetext{
${ }^{1}$ Princeton University, Department of Astrophysical Sciences, Princeton, NJ 08544-1001, USA

${ }^{2}$ Fermi National Accelerator Laboratory, P.O. Box 500, Batavia, IL 60510, USA
} 